\title{
Saturation of coupling of collective levels in optical model calculations of even-even actinides
}

\author{
José Manuel Quesada $^{1}$, Dmitry Martyanov²a, Efrem Soukhovitskiĩ ${ }^{2}$, Roberto Capote ${ }^{3}$, and Satoshi Chiba ${ }^{4}$ \\ 1 Departamento de Física Atómica, Molecular y Nuclear, Universidad de Sevilla, Sevilla, Spain \\ 2 Nuclear Evaluation Lab, Joint Institute for Energy and Nuclear Research, Minsk, Belarus \\ 3 NAPC-Nuclear Data Section, International Atomic Energy Agency, Vienna, Austria \\ ${ }^{4}$ Laboratory for Advanced Nuclear Energy, Institute of Innovative Research, Tokyo Institute of Technology, Japan, and National \\ Astronomical Observatory of Japan, Tokyo, Japan
}

\begin{abstract}
Saturation of the coupling-scheme is studied in dispersive optical model calculations of nucleon induced reactions on ${ }^{238} \mathrm{U}$ target. Recently derived potential that is based on a soft-rotator-model (SRM) description of the collective levels of the target nucleus with volume conservation is used. It is show that calculated direct excitation cross sections of all considered non-GS bands levels are larger than the $4^{+}$GS band level excitation, and can't be ignored in coupled-channel calculations. SRM couplings of 21 levels constitute a saturated coupling scheme that allows a precise calculation of the compound-nucleus $(\mathrm{CN})$ formation cross sections up to several $\mathrm{MeV}$.
\end{abstract}

\section{Introduction}

For a long time it has been believed that in coupledchannels optical model (OM) calculations, the axial rigidrotator coupling scheme would quickly saturate after including very few coupled levels (e.g., see Refs. [1,2]). By saturation we understand the situation in which the addition of higher energy levels to the coupling scheme does not practically change the predicted OM observables. In fact, three first levels have been routinely coupled and used for nuclear data analysis and evaluation. In 2004 some of authors demonstrated that a minimum of 5 coupled levels (up to $8^{+}$state in even-even targets) was needed to saturate calculated total cross sections [3]. In 2012 the saturation of calculated compound-nucleus (CN) formation cross section was studied by Dietrich et al. [4]. They pointed out that in axial rigid-rotator calculations, the saturation of the reaction cross section requires a minimum of 7 coupled levels despite the fact that the predicted inelastic scattering cross sections on the high-spin levels are very small [4]. However, only the saturation of coupled ground-state band levels has been studied so far.

Accurate analysis of low-lying collective levels of even-even actinides indicates their nonaxiality and softness to quadrupole deformations $[5,6]$ Measurements of double differential neutron emission spectra from ${ }^{238} \mathrm{U}$ exhibit significant excitation of levels of these bands [7] with cross sections comparable to predicted $6^{+}$GS-band level excitation. Thus coupling of these collective levels can not be ignored in consistent coupled-channels optical model calculations.

Furthermore, nuclear data for fast reactors require lower uncertainty of estimated inelastic scattering cross sections on ${ }^{238} \mathrm{U}$ nucleus, which represents a major actinide

\footnotetext{
a e-mail: dmart@sosny.bas-net.by
}

component of the reactor fuel [8]. Fast reactor spectra typically extend from a few $\mathrm{keV}$ up to $5-6 \mathrm{MeV}$ to cover the region of maximum yield of fission neutrons. Therefore, low-lying rotational bands built on vibrational bandheads at excitation energies above $500 \mathrm{keV}$ for eveneven targets need to be taken into account. This fact has long been acknowledged, many groups have used DWBA approximations or coupled-channels approaches to describe scattering data on even-even actinides (e.g., by University of Lowell group [9-11], Kawano et al. [12], Minsk group [13,14] and Bruyères-le-Châtel group $[15,16])$. However, these works used non-dispersive potentials to describe scattering data, except the dispersive potential developed by French researchers $[15,16]$.

\section{Optical model description}

We have developed a vibrational-rotational structure model [17-20] which allows coupling of collective levels belonging to different rotational bands in OM calculations of nucleon induced reactions on strongly deformed targets. The coupling model was extended to consider volume conservation and a better structure information by using a soft-rotator model (SRM) [21]. The SRM [6,22] describes very well the energies of low-lying collective states in actinide nuclei as well as $\gamma$-transition probabilities $B(E \lambda)$ between excited levels. SRM and rigid-rotator model predicted excitation energies of the ground state band are compared in Table 1 . One can see that for increasing spins of levels, rigid-rotator energies become higher than experimental or soft-rotator values and the difference increases with level spin indicating a stretching of the rotating nucleus.

The extended optical model using SRM couplings (present model, PM) allowed calculation of "effective" deformations determining channel coupling in optical 
Table 1. ${ }^{238} \mathrm{U}$ level energies: experiment vs soft-rotator and rigid-rotator structure models.

\begin{tabular}{lllll}
\hline Band & $J^{\pi}$ & $E_{\text {exp }}, \mathrm{kev}$ & $E_{\text {soft }}, \mathrm{kev}$ & $E_{\text {rigid }}, \mathrm{kev}$ \\
\hline $\mathrm{GS}$ & $0^{+}$ & 0.0 & 0.0 & 0.0 \\
& $2^{+}$ & 44.92 & 45.54 & 44.92 \\
& $4^{+}$ & 148.38 & 150.14 & 149.72 \\
& $6^{+}$ & 307.18 & 309.95 & 314.41 \\
& $8^{+}$ & 518.10 & 519.47 & 539.00 \\
& $10^{+}$ & 775.90 & 772.23 & 823.46 \\
\hline
\end{tabular}

calculations and was used in this work; it also considers the mixing of states with all possible $K$ values ("with $K$ admixtures") that appears due to assumed intrinsic non-axiality of the nucleus. Here we analyze predictions of the model for the $n+{ }^{238} \mathrm{U}$ reaction coupling almost all observed collective levels of five rotational bands, including a negative parity one (OCT), a non-axial band (NAX), $\beta$ - and $\gamma$ - quadrupole vibrational bands, with excitation energies up to about $1.2 \mathrm{MeV}$ (GS: $I^{\pi}=$ $0^{+} \ldots 10^{+}, \beta: I^{\pi}=0^{+} \ldots 6^{+}, \gamma: I^{\pi}=0^{+} \ldots 4^{+}, \mathrm{NAX}:$ $I^{\pi}=2^{+}, 3^{+}, 4^{+}$, OCT: $I^{\pi}=1^{-} \ldots 9^{-}, 21$ level in total and 3 lowest isobar-analogue states). All OM calculation in this paper used the OPTMAN code $[23,24]$ with dispersive integrals calculated analytically $[20,25,26]$ and full Lane consistency [27].

\section{Results}

Compound nucleus formation $(\mathrm{CN})$ cross section $\sigma_{C N}(E)$ is very hard to measure, but it is a critical quantity for reaction modelling as it determines the cross section that will be distributed by statistical reaction models following the Bohr hypothesis. Figure 1 shows calculated $\mathrm{CN}$ formation cross sections for three different models compared to present model results with full couplings (PM). Note that only the PM considers $K$ mixing and nuclear volume conservation. "21 CC" model $[19,20]$ is a vibrationalrotational optical model with extended coupling of 21 levels and "effective" deformations of vibrational bands used as free parameters of the model. "5 CC" is an axial rigid-rotator model with five GS levels coupled. All three models were fitted to the same experimental data including the direct $(p, n)$ reaction angular distributions with 3 lowest isobaric analog states (IAS) excitation. "2408" potential is an axial rigid-rotator model with five GS band levels coupled developed by Capote et al. [28,29]; the corresponding RIPL database index [30] of this potential is 2408 .

In Fig. 1 we can see that differences between predictions in $\mathrm{CN}$ formation cross section from models which only couple GS band levels and those models that also couple levels of other bands may reach up to $100 \mathrm{mb}$, even if corresponding OM potentials had been adjusted to the same total cross sections and neutron strength functions. In Fig. 2 different non-GS band excitation cross sections (summed over all band levels) are compared with the excitation cross section of GS band levels $4^{+}, 6^{+}$, and $8^{+}$calculated with the present model. One can see that excitation cross sections of the octupolar and $\beta$ bands are larger than the one of the $6^{+}$GS band level. Therefore, for the sake of consistency, if we take into account in the coupling scheme the $6^{+}$GS band level, we also need to include the octupolar and $\beta$-bands. For the same reason,

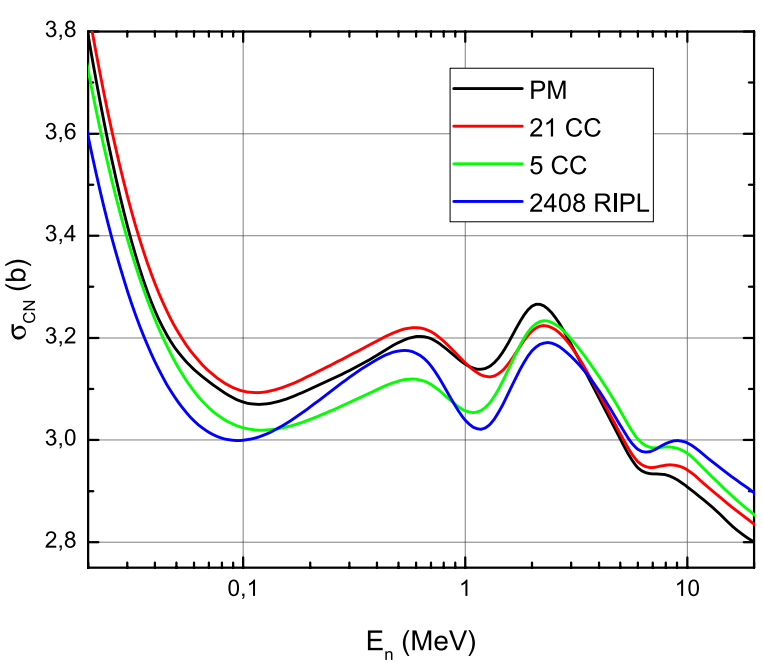

Figure 1. Calculated $\sigma_{C N}(E)$ for $n+{ }^{238} \mathrm{U}$ reaction using rigidand soft-rotator couplings and different coupling schemes.

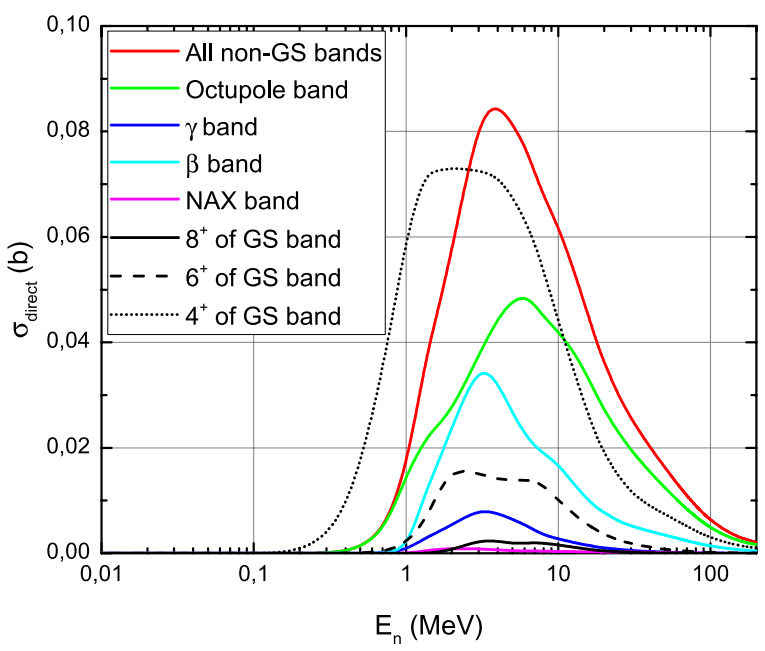

Figure 2. Calculated direct level excitation cross sections for $n+{ }^{238} \mathrm{U}$ reaction using present model with full couplings. NonGS band level cross sections are summed by bands. The red line represents the sum of excitation cross sections of all non-GS levels.

accounting of $8^{+}$GS band level requires the account of all of our considered non-GS bands $(\beta, \gamma, \mathrm{NAX}, \mathrm{OCT})$.

What is the impact on calculated $\mathrm{CN}$ formation cross sections of the softness and non-axiality of the nucleus as considered by the SRM? To isolate the effect of nuclear softness and $K$ mixing on optical predictions we have intentionally limited the coupling scheme to the first 5 levels of the GS band. As a reference case we took the conventional rigid-rotator case (RAP) and fitted the OM parameters and ${ }^{238} \mathrm{U}$ deformations that describe the same experimental database used for other variations. Then, we have used this potential and the same coupling scheme to calculate optical observables with account of nuclear softness and $K$ mixing both separately and simultaneously. An additional calculation used the same potential (rigid case with $K$ mixing) but with coupling scheme extended to include the NAX band. Calculated $\mathrm{CN}$ formation cross section for all five studied cases are shown in Fig. 3 as a ratio to $\mathrm{PM}$ calculated $\mathrm{CN}$ cross section $R=2\left(\sigma_{C N}-\sigma_{C N}^{P M}\right) /\left(\sigma_{C N}+\sigma_{C N}^{P M}\right)$. 


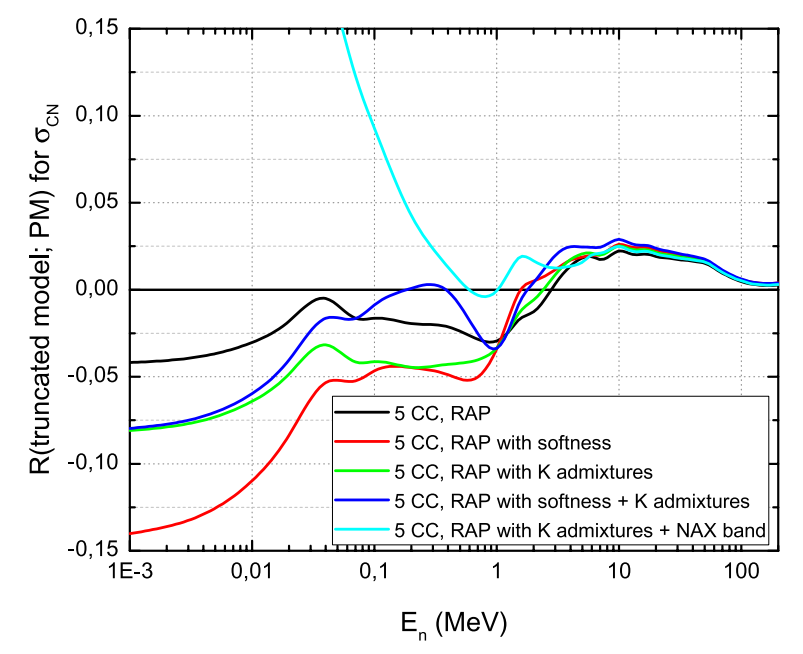

Figure 3. Dependence of the calculated $\sigma_{C N}(E)$ on different model assumptions using five coupled GS levels for $n+{ }^{238} \mathrm{U}$ reaction plotted as a ratio to the $\sigma_{C N}(E)$ calculated with the present model with full couplings.

Differences in calculated cross sections due to nuclear softness reach almost $10 \%$ at $1 \mathrm{keV}$ and decrease above $5 \mathrm{MeV}$. Therefore, nuclear softness is important for optical model calculations at low energies even if only five GS levels are coupled. Additional coupling of vibrational band levels increases the observed differences.

The impact of non-axiality on calculated cross sections is more complex. First of all, collective rotational levels with $I^{\pi}$ other than $0^{+}, 3^{+}$and $1^{-}$have admixtures of wavefunctions corresponding to excited vibrational bands. The $K$-mixing increases with the spin. As seen from Fig. 3, the non-axiality has a similar impact on calculated cross sections as the nuclear softness leading to $4 \%$ difference at $1 \mathrm{keV}$. Inclusion of NAX band levels in coupling scheme changes $\mathrm{CN}$ formation cross section considerably for incident energies below $5 \mathrm{MeV}$.

In the foregoing paragraphs we have shown that neglecting non-axiality and nuclear softness in conventional axial rigid-rotator coupled channel optical model calculations for strongly deformed heavy nuclei (e.g., actinides) lead to non-reliable predictions of optical observables. A present model that combines a SRM description with volume conservation in the dispersive coupled-channels treatment is free of such drawbacks.

PM considers four rotational bands besides the GS band. One can expect that non-GS bands will saturate at lower spins than GS-band since experimentally the measured sum of GS band levels $2^{+}$and $4^{+}$excitations $(\approx 0.45 \mathrm{~b}$ at its maximum around $2 \mathrm{MeV}$ [31]) is about ten times larger than the excitation of the most strongly populated octupolar band $(\approx 0.045 \mathrm{~b}$ at its maximum around $6 \mathrm{MeV}$ ) (see Fig. 2).

We analyzed the impact of the exclusion of the highest spin level of each band included in our PM. Figure 4 shows the ratio of the $\mathrm{CN}$ formation cross sections calculated with truncated (all but one with highest spin in each band) level schemes to the one with the full coupling, in all cases using the PM potential. One can see that calculations with truncated level scheme result in up to $6.5 \%$ cross-section change. The difference caused by neglecting the $10^{+} \mathrm{GS}$ band level is close to the one found by Dietrich et al. [4].

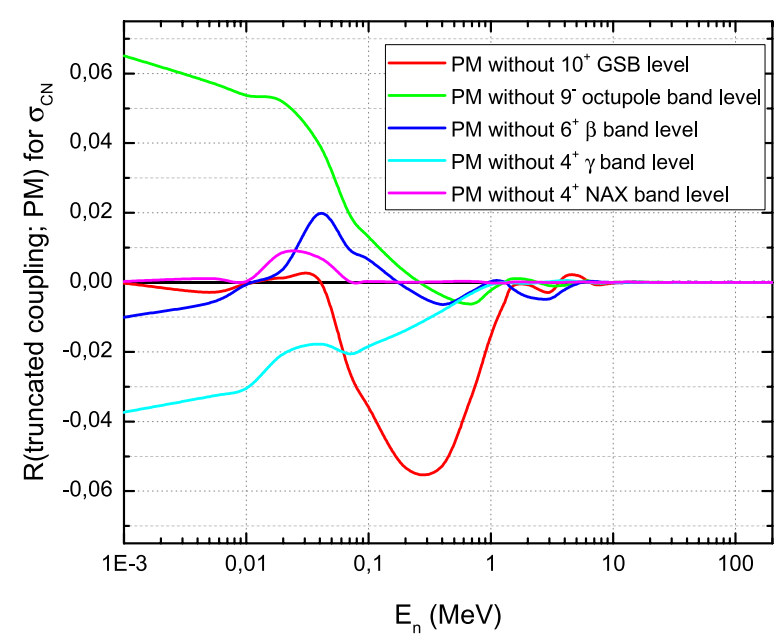

Figure 4. Dependence of the calculated $\sigma_{C N}(E)$ using softrotator couplings on different truncated coupling schemes for the $n+{ }^{238} \mathrm{U}$ reaction plotted as a ratio to the $\sigma_{C N}(E)$ calculated with the present model with full couplings.

Those authors also found that the addition of the $12^{+}$GS band level to their level scheme changes $\mathrm{CN}$ formation cross section by less than $1 \%$. Our calculations predict the same impact for the addition of higher spin levels to each non-GS band (OCT: $I^{\pi}=11^{-}, \gamma: I^{\pi}=6^{+}, \beta: I^{\pi}=8^{+}$, NAX: $\left.I^{\pi}=5^{+}\right)$. Therefore, we can consider that the PM with full coupling represents a saturated coupling scheme.

\section{Conclusions}

Calculations with SRM coupled-channels optical model show that a consistent $\mathrm{CN}$ formation cross section estimation requires the coupling of GS band levels up to $10^{+}$and levels of rotational bands built on octupole, quadrupole $\beta$ - and $\gamma$-vibrational excitations, and nonaxial $(K \approx 2)$ bands. High spin levels having negligible direct excitation cross sections $(<1 \mathrm{mb})$ should nevertheless be included into the coupling scheme to calculate $\mathrm{CN}$ formation cross section with high accuracy. Nuclear softness against quadrupole and octupole shape deformations and accounting of the $K$-mixing due to nonaxiality are important to guarantee the lowest uncertainty of calculated OM cross sections.

This work was partially funded by the Spanish Ministry of Economy and Competitivity under Contract FPA2014-53290-C22-P. One of the authors (D.M.) is grateful to IAEA for financial support that allowed to attend the Conference.

\section{References}

[1] R.C. Barrett, Nucl. Phys. 51, 27 (1964)

[2] E.S. Sukhovitskiũ, O. Iwamoto, S. Chiba, and T. Fukahori, J. Nucl. Sci. Technol. 37, 120 (2000)

[3] E.Sh. Soukhovitskiĩ et al., J. Phys. G: Nucl. Part. Phys. 30, 905 (2004)

[4] F.S. Dietrich, I.J. Thompson, and T. Kawano, Phys. Rev. C85, 044611 (2012)

[5] J.M. Eisenberg and W. Greiner, Nuclear Theory: Nuclear models, (North-Holland, Amsterdam, 1987)

[6] Yu.V. Porodzinkiĩ and E.Sh. Sukhovitskiĩ, Phys. At. Nuclei 59, 228-237 (1996) 
[7] M. Baba, H. Wakabayashi, N. Ito, K. Maeda, and N. Hirakawa J. Nucl. Sci. \& Tech. 27, 601-616 (1990)

[8] R. Capote, A. Trkov, M. Sin, M.W. Herman, and E.Sh. Soukhovitskii, EPJ Web of Conf. 469, 00008 (2014)

[9] D.W.S. Chan, J.J. Egan, A. Mittler, E. Sheldon, Phys. Rev. C26 (1982) 841

[10] D.W.S. Chan and E. Sheldon, Phys. Rev. C26 (1982) 861

[11] E. Sheldon. L.E. Beghian, D.W. Chan et al., J. Phys. G: Nucl. Phys. 12, 443 (1986)

[12] T. Kawano, N. Fujikawa, and Y. Kanda, "Evaluation of ${ }^{238}$ U inelastic scattering cross section", Technical Report JAERI-M-94-019, INDC(JPN)-169, Japan Atomic Energy Research Institute, Ibaraki-ken, Japan (1994)

[13] E.Sh. Sukhovitskiĩ and Yu.V. Porodzinskiñ, Proc. of 9-th Int.Symposium on Capture Gamma-Ray Spectroscopy and Related Topics, Budapest, Hungary, Vol.1, p. 335 (1996)

[14] V.M. Maslov, Yu.V. Porodzinskij, N.A. Tetereva, M. Baba, and A. Hasegawa, Nucl. Phys. A 764, 212-245 (2006)

[15] M.J. Lopez Jimenez, B. Morillon, and P. Romain, Ann. Nucl. Energy 32, 195-213 (2005)

[16] P. Romain. B. Morillon, and H. Duarte, Nucl. Data Sheets 131, 222-258 (2016)

[17] J.M. Quesada, E.Sh. Soukhovitskiĩ, R. Capote, and S. Chiba, presented at WONDER-2012, Aix-auProvence, France, EPJ Web of Conf. 42, 02005 (2013)

[18] J.M. Quesada, R. Capote, E.Sh. Soukhovitskiĩ and S. Chiba, Nucl. Data Sheets 118, 270-272 (2014)

[19] J.M. Quesada, R. Capote, E.Sh. Soukhovitskiĩ and S. Chiba, EPJ Web of Conf. 111, 03004 (2016)
[20] E.Sh. Soukhovitskiĩ, R. Capote, J.M. Quesada, S. Chiba and D. Martyanov, submitted to Phys. Rev., Dec. 2015

[21] D. Martyanov, E. Soukhovitskĩ̃, R. Capote, J.M. Quesada, and S. Chiba, this conference

[22] Yu.V. Porodzinskiĩ and E.Sh. Sukhovitskiĩ, Sov. J. Nucl. Phys. 53, 41-44 (1991)

[23] E.Sh. Soukhovitskĩ̃, S. Chiba, O. Iwamoto, K. Shibata, T. Fukahori, and G.B. Morogovskiĩ, "Programs OPTMAN and SHEMMAN Version 8", Technical report JAERI-Data/Code 2005-002, Japan Atomic Energy Research Institute (2005)

[24] E.Sh. Soukhovitskiũ, G.B. Morogovskiĩ, S. Chiba, O. Iwamoto, and T. Fukahori. "Physics and Numerical Methods of OPTMAN: A Coupledchannels Method Based on Soft-rotator Model for a Description of Collective Nuclear Structure and Excitation". Technical report JAERI-Data/Code 2004-002, Japan Atomic Energy Research Institute (2004)

[25] J.M. Quesada, R. Capote, A. Molina, and M. Lozano, Comp. Phys. Commun. 153, 97-105 (2003)

[26] J.M. Quesada, R. Capote, A. Molina, M. Lozano, and J. Raynal, Phys. Rev. C67, 067601 (2003)

[27] J.M. Quesada, R. Capote, E.Sh. Soukhovitskiĩ, and S. Chiba, Phys. Rev. C76, 057602 (2007)

[28] R. Capote, E. Sh. Soukhovitskiu, J.M. Quesada, and S. Chiba, Phys. Rev. C72, $064610(2005)$

[29] R. Capote, S. Chiba, E.Sh. Soukhovitskiĩ, J.M. Quesada, and E. Bauge, J. Nucl. Sc. Tech. 45, 333-340 (2008)

[30] R. Capote et al., Nucl. Data Sheets 110, 3107-3214 (2009) (see http://www-nds.iaea. org/RIPL-3/)

[31] G. Haouat et al., Nucl. Sci. \& Eng. 81, 491 (1982) 